\title{
Evaluation of Insulin Like Growth Facror-1 Genetic Polymorphism with Gastric Cancer Susceptibility and Clinicopathological Features
}

\author{
Roya Kishani Farahani ${ }^{1}$, Pedram Azimzadeh ${ }^{1}$, Elham Rostami ${ }^{1}$, Habib \\ Malekpour ${ }^{2}$, Hamid Asadzadeh Aghdae', Mohamad Amin Pourhoseingholi², \\ Ehsan Nazemalhosseini Mojarad ${ }^{2 *}$, Mohammad Reza Zali
}

\begin{abstract}
Gastric cancer (GC) is one of the most common malignancies in the world. It is the first cause of cancer deaths in both sexes In Iranian population. Circulating insulin-like growth factor-one (IGF-1) levels have been associated for gastric cancer. IGF-1 protein has central roles involved in the regulation of epithelial cell growth, proliferation, transformation, apoptosis and metastasis. Single nucleotide polymorphism in IGF-1 regulatory elements may lead to alter in IGF-1expression level and GC susceptibility. The aim of this study was to investigate the influence of IGF-1 gene polymorphism (rs5742612) on risk of GC and clinicopathological features for the first time in Iranian population. In total, 241 subjects including 100 patients with GC and 141 healthy controls were recruited in our study. Genotypes were analyzed using polymerase chain reaction-restriction fragment length polymorphism (PCR-RFLP) assay with DNA from peripheral blood. The polymorphism was statistically analyzed to investigate the relationship with the risk of GC and clinicopathological properties. Logistic regression analysis revealed that there was no significant association between rs5742612 and the risk of GC. In addition, no significant association between genotypes and clinicopathological features was observed ( $p$ value $>0.05$ ). The frequencies of the CC, CT, and TT genotypes were $97 \%, 3 \%$, and $0 \%$, respectively, among the cases, and $97.9 \%$, $2.1 \%$, and $0 \%$, respectively, among the controls. $\mathrm{CC}$ genotype was more frequent in cases and controls. The frequencies of $\mathrm{C}$ and $\mathrm{T}$ alleles were $98.9 \%$ and $1.1 \%$ in controls and $98.5 \%$ and $1.5 \%$ in patient respectively. Our results provide the first evidence that this variant is rare in Iranian population and it may not be a powerful genetic predisposing biomarker for prediction GC clinicopathological features in an Iranian population.
\end{abstract}

Keywords: Gastric cancer - genetic polymorphism - IGF-I - clinicopathological features

Asian Pac J Cancer Prev, 16 (10), 4215-4218

\section{Introduction}

Gastric cancer (GC) is the fifth most commonly occurring cancer and third leading cause of cancer mortality in the world (McLean and El-Omar, 2014). In Iranian population, GC is the first cause of cancer deaths in both sexes (Smith et al., 2006; Malekzadeh et al 2009). The etiology of GC is not clearly known. Several risk factors include environmental agents (diet, smoking); infectious aspects (Helicobacter pylori) and genetic factors are suspected to play a significant role in gastric carcinogenesis (Katoh et al., 1996; Stadtlander 1999; Setiawan et al., 2000; Forman and Burley, 2006; Rostami et al., 2011). Among the some genetic changes, there is evidence that the upregulation of certain growth factors could play a significant role in the development and progression of gastric cancer (Altinkaynak et al., 2012). Insulin-like growth factors-1 (IGF-1) is one of the significant growth factors. It is multifunctional peptide, affecting cell proliferation in almost every cell type through the RAS, RAF-MAP kinase signaling pathway and also has antiapoptotic influence via activation of the phosphatidylinositol-3 kinase-AKT pathway, which use activates downstream transcription factors that mediate gene expression of proliferation, differentiation, and antiapoptotic agents (Karamouzis and Papavassiliou, 2006; Samani et al., 2007; Tao et al., 2007; Pollak 2008; Yang et al., 2014). High levels of circulating IGF-1 are relation to increase risk of several common cancers such as prostate, colorectal, breast, lung, glioma and gastric cancer (Yu and Rohan, 2000; Furstenberger and Senn, 2002; Lacey et al., 2004; Chen et al., 2009; Shitara et al., 2011; Altinkaynak et al.,2012; Lian et al., 2014). The levels of circulating IGF-1 are highly influenced by genetic factors (Harrela et al., 1996). Although there is a few researches have investigated the important of IGF-I polymorphisms and susceptibility or progression to types of cancers (Gao et al., 2009). According to our knowledge there is only one

${ }^{1}$ Basic and Molecular Epidemiology of Gastroenterology, ${ }^{2}$ Gastroenterology and Liver, Disease Research Center, Shahid Beheshti University of Medical Sciences, Tehran, Iran*For correspondence: ehsanmojarad@gmail.com 


\section{Roya Kishani Farahani et al}

data about this polymorphism and gastric cancer (Shitara et al., 2011). To address this issue we select rs5742612 in promoter region which is influence to IGF-1 level to identify its susceptibility and progression effects on GC in Iranian population (D'Aloisio et al., 2009). We want to determine its power potential predisposing as a biomarker for GC prognosis in our population. We also aim to find association of this polymorphism with clinicpathological and demographic features.

\section{Materials and Methods}

\section{Patients and controls}

The IGF-1 polymorphism was evaluated by a casecontrol study that was conducted on a population of 100 patients suffering from Gastric cancer and 141 normal individuals who had been referred to the Research Institute for Gastroenterology and Liver Diseases (RIGLD), Taleghani Hospital, Shahid Beheshti University of Medical Sciences (Tehran, Iran). Studied Subjects were Iranian and before taking blood sample of individuals was obtained consent informed. Patients had pathology and clinical symptoms that were indicative of gastric cancer and controls had no this Symptoms. This study was conducted under the approval of the ethics committee of the gastroenterology and liver disease research center, Shahid Beheshti University of medical sciences (Tehran, Iran).

\section{Selection and evaluation of genetic polymorphisms}

Polymorphism rs5742612 is selected based on: i) pervious research finding who found association of rs5742612 with $10 \%$ or greater difference in IGF-1 level ii) some papers showed this variant significantly associated with various types of malignancies iii) as far as we know, there has been just only one research about the association between the rs5742612 and susceptibility or progression of gastric cancer in the literature so far (Shitara et al., 2011).

\section{DNA extraction:}

$5 \mathrm{ml}$ peripheral blood was collected and stored at $4^{\circ} \mathrm{C}$ in a bottle containing EDTA. Genomic DNA was extracted as soon as possible following sampling using the standard salting out method (Miller et al., 1998). The quality of the extracted DNA was then assessed using a NanoDrop spectrophotometer (NanoDrop Technologies, Inc., Wilmington, DE, USA). Samples were frozen at $-20^{\circ} \mathrm{C}$ until further analysis.

\section{IGF-1 rs5742612 gene polymorphism genotyping}

The PCR restriction fragment length polymorphism assay was used to detect the IGF-1 polymorphisms. The 410 bp DNA fragment of the IGF-1 gene was amplified using the specific primers (forward 5':GACAGTGACAGGCAGCCTAGT 3' and reverse 5': CTGGGCATGAAGACACAAACG 3') which were designed based on IGF-1 gene were used for PCR. PCR condition and program was the initial denaturation was carried out at $94^{\circ} \mathrm{C}$ for $10 \mathrm{~min}$, then, the reaction was as follows: 33 cycles of $95^{\circ} \mathrm{C}$ for $45 \mathrm{sec}, 63^{\circ} \mathrm{C}$ for $40 \mathrm{sec}$, $72^{\circ} \mathrm{C}$ for $40 \mathrm{sec}$, followed by a final extension at $72^{\circ} \mathrm{C}$ for $10 \mathrm{~min}$. The PCR products were digested by BselI endonuclease enzyme in $55^{\circ} \mathrm{C}$ at overnight. In order to observe the digested fragments, the RFLP solution was sepa $\neg$ rated on a $3 \%$ agarose gel and stained with ethidium bromide.

\section{Statistical analysis}

Hardy-Weinberg equilibrium (HWE) was tested using a goodness-of-fit $\chi^{2}$ test. For estimate adjusted and unadjusted odds ratio (OR) Unconditional logistic regression analysis was performed and 95\%confidence interval (CI) as a major of association of the genotypes with the risk of gastric cancer. OR and $95 \%$ CI were adjusted for age and sex state. By chi-square test relation between genotype and clinic pathology were examined in gastric cancer patients.

\section{Results}

In the present study, we evaluated effect of polymorphisms rs5742612 at insulin like growth factor -1 gene with risk of gastric cancer. The frequency distribution of selected characteristics of 100 colorectal cancer patients and 141 healthy controls are shown in Table 1 . Ages ranged from 37-82 years for cases and 19-92 years for controls. The mean age of GC patients and controls respectively were 48.46 (Standard deviation, $\mathrm{SD} \pm 16.63$ ) and $63.66(\mathrm{SD} \pm 12.39)$ years. There were no significant differences between the GC cases and controls in the terms of diabetes, hypertension and $\mathrm{BMI}(\mathrm{P}=0.27, \mathrm{P}=0.51$, $\mathrm{P}=0.1$ respectively). However, all of these variables were adjusted further for any residual confounding effect in later multivariate logistic regression analyses. The genotype and allele frequencies of IGF-1 polymorphisms rs5742612 for the controls and the cases are shown in Table 2. The frequencies of the CC, CT, and TT genotypes were $97 \%$, $3 \%$, and $0 \%$, respectively, among the cases, and $97.9 \%$, $2.1 \%$, and $0 \%$, respectively, among the controls. Genotype distributions in controls and cases were consistent with the Hardy-Weinberg equilibrium (HWE). Our results showed CC genotype was the most genotype in controls and patients in comparison to other genotypes. Also TC genotype was similar in healthy controls and gastric cancer patients $(\mathrm{p}=0.67)$ and we did not find any TT genotype in all of the case and control groups. The frequencies of $\mathrm{C}$ and $\mathrm{T}$ alleles were $98.9 \%$ and $1.1 \%$ in controls and $98.5 \%$ and $1.5 \%$ in patient respectively. Statistical analysis revealed no significant difference in the allele frequency between case and control individuals $(\mathrm{p}=0.672)$. In this SNPs analyzed, the P value is larger than 0.05 . In addition to these, the distributions of clinicopathologic variables such as grade, TNM, are shown in Table 3. According to histological differentiation of tumor grades, 17\%, 9\%, and $12 \%$, patients were classified in poor, moderate, or well grade, respectively. Tumor grade distribution was such that poor grade status was more common (17\%) than others. Besides, patients were grouped into four classes from I to IV regarding tumor node metastasis (TNM) at the time of diagnosis, $4 \%, 8 \%, 22 \%$, and $8 \%$ respectively. No significant association was found between rs5742612 of IGF-1 gene and risk of gastric cancer (Table 2). 
Insulin Like Growth Facror-1 Genetic Polymorphism in Gastric Cancer Susceptibility and Clinicopathological Features

Table 1. Characteristics of Gastric cancer Patient and Control Groups

\begin{tabular}{|c|c|c|c|c|c|c|c|}
\hline \multirow[t]{2}{*}{ Characteristics } & \multicolumn{3}{|c|}{ Patients $(n=100)$} & \multicolumn{3}{|c|}{ Controls $(\mathrm{n}=141)$} & \multirow[t]{2}{*}{$\mathrm{P}^{\mathrm{a}}$} \\
\hline & $\mathrm{N}$ & $\%$ & $\operatorname{Mean}(\mathrm{SD})$ & $\mathrm{N}$ & $\%$ & $\operatorname{Mean}(\mathrm{SD})$ & \\
\hline BMI & & & $24.60 \pm 3.63$ & & & $21.07 \pm 2.99$ & 0.1 \\
\hline Diabet & & & & & & & 0.2 \\
\hline Yes & 4 & 44.4 & & 5 & 55.6 & & \\
\hline No & 47 & 27.5 & & 124 & 72.5 & & \\
\hline Blood pressure & & & & & & & 0.51 \\
\hline Yes & 2 & 16.7 & & 10 & 83.3 & & \\
\hline No & 49 & 29.2 & & 119 & 70.8 & & \\
\hline
\end{tabular}

Table 2. Allele and Genotype Distribution of rs5742612 among GC Patients and Healthy Control Subjects

\begin{tabular}{|c|c|c|c|c|}
\hline Genotypes & $\begin{array}{c}\text { Controls } \\
\mathrm{n}=141(\%)\end{array}$ & $\begin{array}{c}\text { Gastric patients } \\
\mathrm{n}=100(\%)\end{array}$ & $\begin{array}{c}\text { Adjusted OR } \\
(95 \% \mathrm{CI})\end{array}$ & $\mathrm{p}$ \\
\hline $\mathrm{CC}$ & $3(50)$ & $3(50)$ & $1 \operatorname{Ref}$ & 0.67 \\
\hline $\mathrm{TC}$ & $138(58.7)$ & $97(41.3)$ & $\begin{array}{l}1.423 \\
(0.281-7.198)\end{array}$ & \\
\hline $\begin{array}{l}\text { Allele } \\
\text { C }\end{array}$ & 297 (98.9) & $197(98.5)$ & $1 \operatorname{Ref}$ & 0.672 \\
\hline $\mathrm{T}$ & $3 \quad(1.1)$ & 3 (1.5) & $\begin{array}{l}0.706 \\
(0.141-3.535)\end{array}$ & \\
\hline
\end{tabular}

Table 3. Association between IGF-1 Genotypes and Clinicopathological Characteristics

\begin{tabular}{lrrrrr}
\hline Characteristics & N(patients=100) & $\%$ & \multicolumn{2}{c}{ Genotype } & P \\
\cline { 5 - 6 } & & & CC & tc & \\
\hline Tumor Grade & & & & & 0.79 \\
Well & 12 & $12 \%$ & 11 & 1 & \\
Moderate & 9 & $9 \%$ & 9 & 0 & \\
Poor & 17 & $17 \%$ & 16 & 1 & \\
Not differentiation & 12 & $12 \%$ & 12 & 0 & \\
unknown & 50 & $50 \%$ & 49 & 1 & \\
TNM stage & & & & & 0.59 \\
I & 4 & $4 \%$ & 4 & 0 & \\
II & 8 & $8 \%$ & 8 & 0 & \\
III & 22 & $22 \%$ & 22 & 0 & \\
IV & 8 & $8 \%$ & 8 & 0 & \\
Unknown & 58 & $58 \%$ & 55 & 3 & \\
\hline
\end{tabular}

\section{Discussion}

In order to find potential predisposing risk factors of $\mathrm{GC}$, we selected an important polymorphism of IGF-1 gene and investigated whether the rs5742612 variant could have an effect on susceptibility and progression of $\mathrm{GC}$ risk in an Iranian population. We also investigated the association of this polymorphism with clinicopathological features include of TNM stage, tumor grade and demographic feathers such as diabetes, blood pressure, ethnicity and body mass index (BMI). We found, for the first time, that variant genotype of rs5742612 were not associated with risk of GC. It is well-known that IGF-1 has significant roles involved in the regulation of epithelial cell growth, proliferation, transformation, apoptosis and metastasis (Delafontaine et al., 2004). The majority of studies investigation correlation between circulating levels of IGF-1 and risk of cancers, but the association of IGF-1 single nucleotide polymorphism with cancer risk has been limited. IGF-1 polymorphisms and elevated serum levels of IGF-1 have been associated with an increased risk of several cancers, including prostate (Wolk et al., 1989; Cheng et al., 2006; Chan et., 2009; Tong et al., 2010), colorectal (Ma et al., 1999; Feik et al., 2010), ovarian (Lukanova et al., 2002; Lukanova et al., 2003) and breast (Setiawan et al., 2006). In some papers showed Polymorphism rs5742612 significantly associated with various types of malignancies (Nakao et al., 2011; Qian et al., 2014). The functional consequence of SNP at rs5742612 is still unclear. Although this variant does not cause any amino acid changes itself, it may have a regulatory function or may be rs 5742612 variation linkage disequilibrium with other functional polymorphisms that is lead to alter in IGF-1 expression level. This is supported by previous report, which showed that genetic variants of rs5742612 were associated with $10 \%$ or greater difference in IGF-1 level (D'Aloisio et al., 2009). According to our knowledge there has been just only one research about the association between the rs5742612 and susceptibility or progression of gastric cancer in the literature so far (Miller et al., 1998). In 2014, Qian and et al. (2014) show there were associated with an elevated risk of Prostate cancer and rs5742612 polymorphism. In a study of Nakao and colleagues conducted on the relation between rs5742612 and pancreatic cancer, they found association of rs5742612 polymorphism and risk of pancreatic cancer among over-weight subjects (Nakao et al., 2011). Other studies showed that inconsistent relationships of this SNP and colorectal cancer such as Arkani and colleagues reported that no evidence of an association between rs5742612 and colorectal cancer (Arkani et al., 2012) but Wong and et al. in 2005 show a significant association between rs5742612 and colorectal cancer (Wong et al., 2005). Whereas in a study in the Germany did not found any significant relation between IGF-1 variant and colorectal cancer risk (Tsugane and Inoue, 2010). In line with a previous study in Iranian population, we found $\mathrm{CC}$ genotype is dominant and there is a low frequency of risk allele in our population (Arkani et al., 2012). We also investigated the association of this polymorphism with clinicopathological and demographic feathers but because of a low frequency of mutant allele there were not significant differences in the genotype distribution and allele frequencies between the patients and control groups of the all putative parameters. The significance of our findings is that in contrast to other Asian populations which have the higher frequency of risk allele; in Iranian population risk allele is a rare (Nakao et al., 2011; Shitara et al., 2011; Qian et al., 2014). Our finding might indicate the peculiar genetic profile of Iranian population in comparison to other population. In conclusion, according to our research, IGF-1 rs5742612 C/T is not associated with the lifestyle factors, susceptibility and progression of gastric cancer in Iranian population, which is the first data for the contribution of the IGF-1 gene in gastric cancer. The evidence from our data and from previous report (Arkani et al., 2012) suggested that rs5742612 variant is uncommon in Iranian population and it may not be a powerful genetic predisposing biomarker for prediction GC clinicopathological features. It is more beneficial to identify other genetic variants in IGF-1 gene especially 


\section{Roya Kishani Farahani et al}

in regulatory regions .However, further studies with sufficiently larger population are required to validate this result and clarify the unsolved issue.

\section{References}

Altinkaynak K, Bilici M, Bakan N, Akcay F (2012). Circulating levels of IGF-I and IGFBP-3 in gastric cancer. Turk $\mathrm{J} \mathrm{Med}$ Sci, 42, 1458-62.

Arkani M, Safaei A, Karimi Kh, Mohsen Vahedi, et al (2012). Association of IGF-1 gene (rs5742612) polymorphism with colorectal cancer. J Sabzevar Univers Med Sc, 19, 109-15.

Altinkaynak K, Bilici M, Bakan N, Akay F (2012). Circulating levels of IGF-I and IGFBP-3 in gastric cancer. Turk $\mathrm{J}$ Med Sci, 42, 1458-62.

Chan JM, Stampfer MJ, Ma J, et al (2002). Insulin-like growth factor-I (IGF-I) and IGF binding protein-3 as predictors of advanced-stage prostate cancer. $J$ Natl Cancer Inst, 94, 1099-106.

Cheng I, Stram DO, Penney KL, et al (2006). Common genetic variation in IGF1 and prostate cancer risk in the multiethnic cohort. J Natl Cancer Inst, 98 ,123-34.

Chen W, Wang S, Tian T, et al (2009). Phenotypes and genotypes of insulin-like growth factor 1, IGF-binding protein-3 and cancer risk: evidence from 96 studies. European J Human Genet, 17, 1668-75.

Delafontaine P, Song Y, Li Y (2004). Expression, Regulation, and Function of IGF-1, IGF-1R, and IGF-1 Binding Proteins in Blood Vessels. Arterioscler Thromb Vasc Biol, 24, 435-44.

D'Aloisio AA, JC Schroeder, Kari E, et al (2009). IGF-I and IGFBP-3 polymorphisms in relation to circulating levels among African American and Caucasian women. Cancer Epidemiol Biomarkers Prev, 18, 954-66.

Feik E, Baierl A, Hieger B, et al (2010). Association of IGF1 and IGFBP3 polymorphisms with colorectal polyps and colorectal cancer risk. Cancer Causes Control, 21, 91-7.

Forman D, Burley V (2006). Gastric cancer: global pattern of the disease and an overview of environmental risk factors. Best Pract Res Clin Gastroenterol, 20, 633-49.

Furstenberger G, Senn HJ (2002). Insulin-like growth factors and cancer. Lancet Oncol, 3, 298-302.

Gao L, Nieters A, Brenner H (2009). Cell proliferation-related genetic polymorphisms and gastric cancer risk: systematic review and meta-analysis. European J Human Genet, 17, 1658-67.

Harrela M, Koistinen H, Kaprio J, et al (1996) Genetic and environmental components of interindividual variation in circulating levels of IGF-1, IGF-II, IGFBP-3. J Clin Invest, 98, 2612-15.

Karamouzis MV, Papavassiliou AG (2006). The IGF-1 network in lung carcinoma therapeutics. Trends Mol Med, 12, 595-602.

Katoh T, Nagata N, Kuroda Y, et al (1996). Glutathione S-transferase M1(GSTM1) and T1 (GSTT1) genetic polymorphism and susceptibility to gastric and colorectal adenocarcinoma. Carcinogenesis, 17, 1855-9.

Lukanova A, Lundin E, Toniolo P, et al (2002). Circulating levels of insulin-like growth factor-I and risk of ovarian cancer. Int J Cancer, 101, 549-54.

Lukanova A, Lundin E, Micheli A, et al (2003). Risk of ovarian cancer in relation to prediagnostic levels of C-peptide, insulinlike growth factor binding proteins-1 and -2 (USA, Sweden, Italy). Cancer Causes Control, 14, 285-92.

Lacey JV, Potischman N, Madigan MP, et al (2004). Insulin-like growth factors, insulin-like growth factor-binding proteins, and endometrial cancer in postmenopausal women: results from a US case-control study. Cancer Epidemiol Biomarkers Prev, 13, 607-12.

Lian H, Zhou Y, Jian Z, Liu R (2014). MiR-323-5p acts as a tumor suppressor by targeting the insulin-like growth factor
1 receptor in human glioma cells. Asian Pac J Cancer Prev, 15, 10181-85.

McLean MH, El-Omar EM (2014). Genetics of gastric cancer. Nature Rev Gastroenterol Hepatol, 11, 664-74.

Malekzadeh R, Derakhshan MH, Malekzadeh Z (2009). Gastric cancer in Iran: epidemiology and risk factors. Arch Iran Med, 12, 576-83.

Miller S, Dykes D, Polesky H (1988). A simple salting out procedure for extracting DNA from human nucleated cells. Nucleic Acids Res, 16, 1215.

Ma J, Pollak MN, Giovannucci E, et al (1999). Prospective study of colorectal cancer risk in men and plasma levels of insulinlike growth factor (IGF)-I and IGFbinding protein-3. J Natl Cancer Inst, 91, 620-5.

Nakao M, Hosono S , Ito H , et al (2011). Interaction between IGF-1 polymorphisms and overweight for the risk of pancreatic cancer in Japanese. Int J Mol Epidemiol Genet, 2, 354-66.

Pollak M (2008). Insulin and insulin-like growth factor signalling in neoplasia. Nat Rev Cancer, 8, 915-28.

Qian J, Zhou H, Chen J, et al (2014). Genetic polymorphisms in IGF-I and IGFBP-3 are associated with prostate cancer in the Chinese population. Plos one, 9, 85609-15.

Rostami M, Kadivar M, Aznab M, Abachi M (2011). Influence of age and gender on association between-765G $>$ C COX-2 genetic polymorphism and gastric adenocarcinoma risk: a case-control study in Iran. Gastroenterol Hepatol Bed Bench, 5, 29-3.

Smith MG, Hold GL, Tahara E, El-Omar EM (2006). Cellular and molecular aspects of gastric cancer. World J Gastroenterol, 12, 2979-90.

Setiawan VW, Zhang ZF, Yu GP, et al (2000). GSTT1 and GSTM1 null genotypes and the risk of gastric cancer: a case-control study in a Chinese population. Cancer Epidemiol Biomarkers Prev, 9, 73-80.

Stadtlander CTH (1999). Molecular epidemiology, pathogenesis and prevention of gastric cancer. Carcinogenesis, 20, 2195208.

Samani AA, Yakar S, LeRoith D, et al (2007). The role of the IGF system in cancer growth and metastasis: overview and recent insights. Endocr Rev, 28, 20-47.

Shitara K, Ito S, Misawa K, et al (2011). Genetic polymorphism of IGF-I predicts recurrence in patients with gastric cancer who have undergone curative gastrectomy. Ann Oncol, 23, 659-64.

Setiawan VW, Cheng I, Stram DO, et al (2006). Igf-I genetic variation and breast cancer: the multiethnic cohort. Cancer Epidemiol Biomarkers Prev, 15, 172-4.

Tong D, Wen X, Jin Y, et al (2010). Changes of androgen receptor and insulin-like growth factor-1 in LNCaP prostate cancer cells treated with sex hormones and flutamide. Asian Pac J Cancer Prev, 11, 1805-9.

Tsugane S, Inoue M (2010). Insulin resistance and cancer: epidemiological evidence. Cancer Sci, 101, 1073-79.

Tao Y, Pinzi V, Bourhis J, Deutsch E (2007). Mechanisms of disease: signaling of the insulin-like growth factor 1 receptor pathway-therapeutic perspectives in cancer. Nat Clin Pract Oncol, 4, 591-602.

Wong HL, Delellis K, Probst-Hensch N, et al (2005). A new single nucleotide polymorphism in the insulin-like growth factor I regulatory region associates with colorectal cancer risk in Singapore chinese. Cancer Epidemiol Biomarkers Prev, 14, 144-51.

Wolk A, Mantzoros CS, Andersson SO, et al (1998). Insulin-like growth factor 1 and prostate cancer risk: a population-based, case-control study. J Natl Cancer Inst, 90, 911-5.

Yu H, Rohan T (2000). Role of the insulin-like growth factor family in cancer development and progression. J Natl Cancer Inst J, 92, 1472-89.

Yang H, Qu R, Lin X , et al (2014). IGF-1 from adipose-derived mesenchymal stem cells promoes radioresistance of breast cancer cells. Asian Pac J Cancer Prev, 15, 10115-9. 